\begin{tabular}{|c|c|c|c|}
\hline Diagnosis & Age & Neurovascular & Aorta $(\mathrm{cm})$ \\
\hline None made & 69 & $\begin{array}{l}\text { Tortuous, dilated vertebral and } \\
\text { carotid arteries }\end{array}$ & Dilated TS 2.9 AA 3.8 \\
\hline $\begin{array}{l}\text { Classic EDS (COL1A1 } \\
\text { mutation) }\end{array}$ & 53 & Bilateral ICA ectasia & Normal TS 3.4 AA 3.1 \\
\hline Clinically vEDS & 32 & Right ICA dissection & Imaging awaited \\
\hline None made & 35 & Left ICA dissection & $\begin{array}{l}\text { Normal dimensions, effaced } \\
\text { contour Ts } 2.9\end{array}$ \\
\hline None made & 48 & Right ICA dissection & $\begin{array}{l}\text { Normal dimensions, effaced } \\
\text { contour TS } 2.7\end{array}$ \\
\hline
\end{tabular}

Background Stroke is a leading cause of disability, but in up to $40 \%$ of cases no cause is found. Although a patent foramen ovale (PFO) is an attractive mechanism to explain these cryptogenic strokes, using current imaging techniques, distinguishing between a causative rather than an incidental PFO remains elusive. We hypothesised that, in the presence of a PFO, atypical right atrial (RA) flow patterns would be linked to embolism risk by increasing the shunt of blood, and as such thrombus through the PFO. In order to investigate this we assessed RA flow patterns and interatrial shunt size in patients with a PFO and investigated whether these metrics predicted the incidence of paradoxical embolism.

Methods 3 groups were recruited; 1) participants with a PFO but no embolism ( $\mathrm{n}=12), 2)$ patients with presumed paradoxical embolism $(n=20)$ and 3 ) participants without a PFO ( $\mathrm{n}=28$ ). All underwent RA 4D flow assessment, and bubble transthoracic echocardiography to determine interatrial shunt size. Atypical RA flow was defined as any flow pattern that was not a classical vortex.

Results

Flow Patterns RA flow patterns were similar between the 2 groups with no embolic event irrespective of the presence of a PFO. In the PFO with embolism group, they were significantly different with a higher incidence of atypical RA flow ( $P$ $=0.0067$, Figure 1B).

Risk of embolism When considering all the subjects with a PFO ( $\mathrm{n}=32$ ), the presence of an atypical flow pattern was 11.5 times more common in those who have had an embolic event $(P=0.002$, Fisher's exact test). To explore whether this effect was mediated by changing the degree of shunting, moderated multiple regression was performed. This showed that flow patterns were related to shunt grade (a pathway, $\beta$ $34.0, \mathrm{p}<0.01$ ), and that shunt grade was related to embolism incidence ( $b$ pathway, $\beta 0.08, p<0.01$ ). As the $a$ and $b$ pathways were significant, mediation analysis was tested using 2,000 bootstrap resamples to generate a 95\% confidence interval (bias corrected) of the indirect effect. This showed that the effect of an atypical flow pattern upon an embolic event is indeed mediated by increasing the shunt across the PFO (CI $0.45-18.42$, Figure 2). As the direct effect of flow patterns on embolic risk becomes insignificant ( 1 pathway, $\mathrm{p}=0.06$ ) this suggests full mediation.

Conclusions Patients with a PFO and atypical RA flow pattern were 11.5 times more likely to have had an embolic event. This increased embolic risk seems to be mediated via increasing the shunt size across the PFO. As a result, not only will identification of the presence or absence of RA classical vortical flow in individuals presenting with a cryptogenic stroke help distinguish a causative PFO, but it may also identify patients with a PFO who are at elevated risk of future embolism.

\section{ATRIAL FIBRILLATION IS ASSOCIATED WITH LEFT VENTRICULAR DYSFUNCTION AND IMPAIRED MYOCARDIAL ENERGETICS THAT PERSIST DESPITE CATHETER ABLATION: IS ATRIAL FIBRILLATION THE CHICKEN OR THE EGG?}

Rohan Wijesurendra*, Alexander Liu, Christian Eichhorn, Eylem Levelt, Rina Ariga, Will Clarke, Christopher Rodgers, Yaver Bashir, Matthew Ginks, Kim Rajappan, Timothy Betts, Vanessa Ferreira, Stefan Neubauer, Barbara Casadei. University of Oxford: *Presenting Author

\subsection{6/heartjnl-2016-309890.138}

Introduction Atrial fibrillation (AF) is associated with increased risk of heart failure and premature death, and is often resistant to treatment. Animal models of pacing-induced AF indicate that AF-induced endothelial dysfunction, impaired coronary reserve, and myocardial remodelling are important in arrhythmia maintenance. However, human AF may reflect a subclinical cardiomyopathy, which persists after sinus rhythm (SR) restoration and provides a substrate for AF recurrence. To test this hypothesis, we investigated the effect of restoring SR by catheter ablation on left ventricular (LV) function and energetics.

Methods 96 subjects were recruited: 53 patients referred for AF ablation, 18 controls in SR matched to patients for age, sex and BMI, and 25 healthy controls (Table 1). Patients had symptomatic paroxysmal $(n=27)$ or persistent $(n=26)$ 'lone' AF (without coronary artery disease, valvular disease, diabetes, uncontrolled hypertension or inadequate ventricular rate-control). Cardiac magnetic resonance (MR) short axis cines were analysed by a blinded investigator to calculate LV volumes and ejection fraction (EF). ${ }^{31}$ Phosphorus MR spectroscopy determined myocardial energetics (ratio of phosphocreatine to ATP [PCr/ATP]). Patients were scanned pre-ablation, early post-ablation $(\sim 20 \mathrm{~h}, \mathrm{n}=48)$ and late post-ablation $(\sim 7$ months, $\mathrm{n}=41)$.
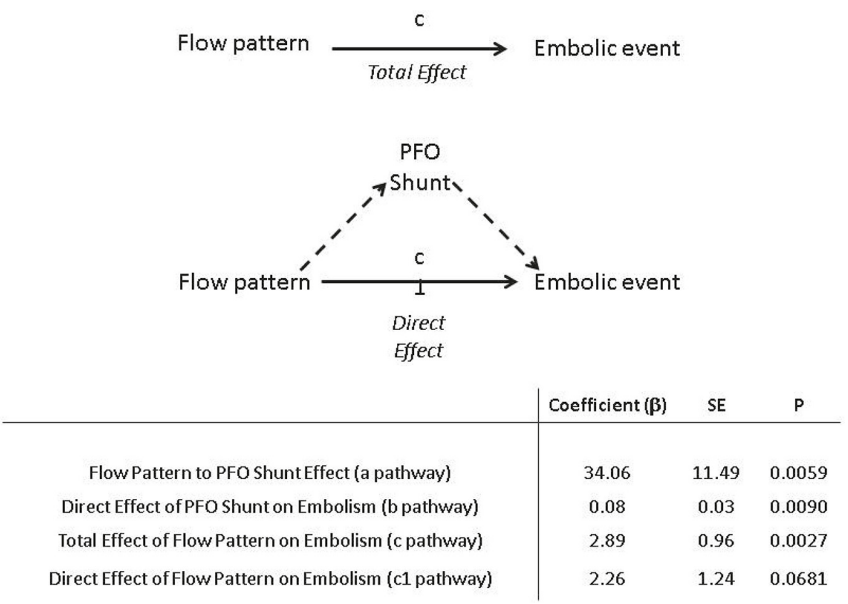

Abstract 137 Figure 2 Mechanistic model showing the contribution of an atypical RA blood flow pattern upon the PFO shunt severity and embolic event occurrence 

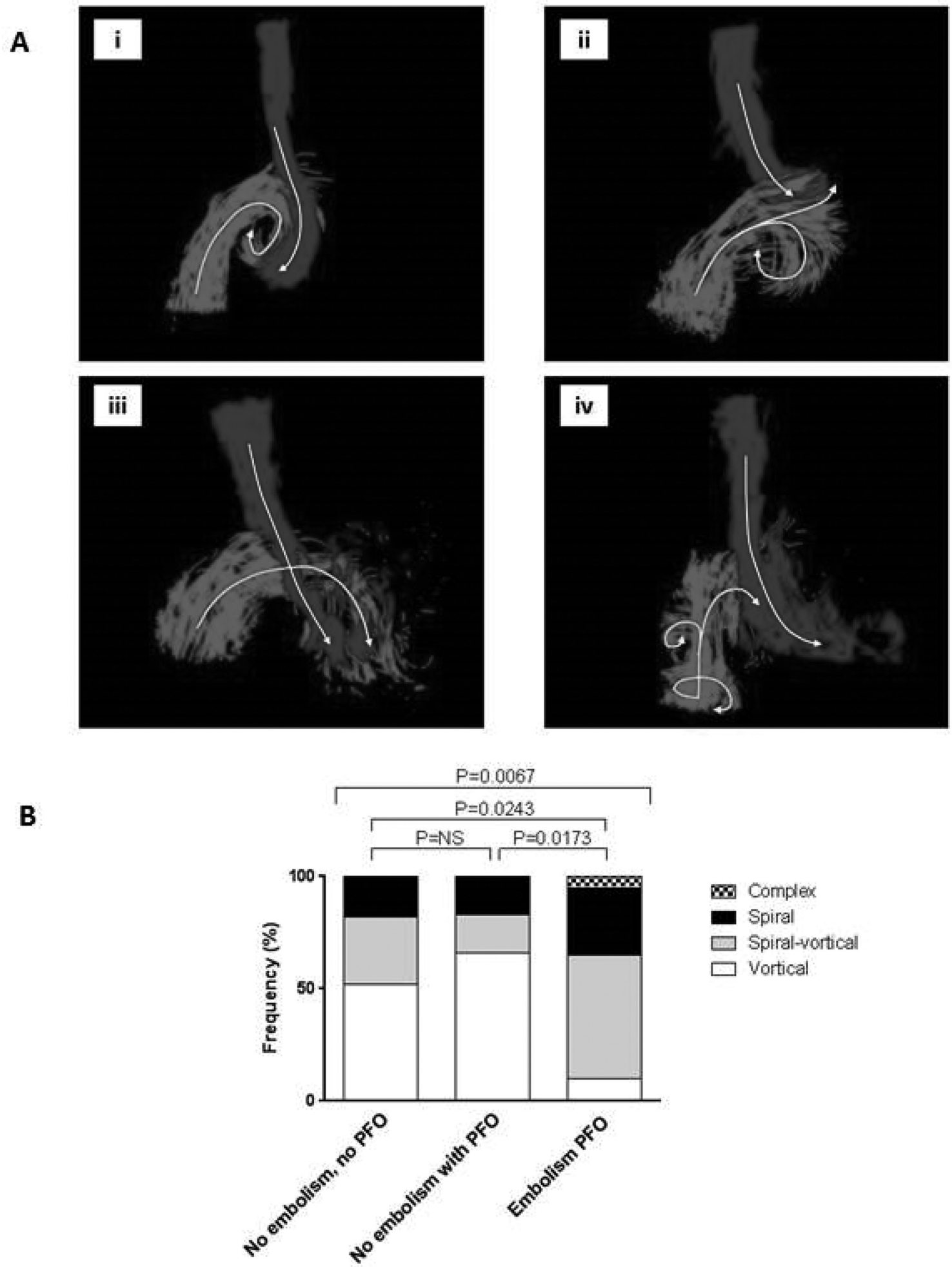

Abstract 137 Figure 1 (A)- i) Vortical flow: the IVC and SVC turn in a clockwise vortex. All other flow patterns were classified as atypical flow patterns: ii) Spiral-vortical flow: the IVC forms a vortex whilst the SVC passes laterally and is then enveloped in a spiral fashion by the IVC, iii)

Results In patients pre-ablation, LVEF was significantly lower than in matched and healthy controls $(59+/ - \pm 10$ vs $69 \pm 6$ and $68 \pm 5 \%$ respectively, $\mathrm{p}<0.001)$. Myocardial energetics was also impaired compared to healthy controls (median $\mathrm{PCr} /$ ATP 1.75 vs 2.06, p $=0.04$ ) but not matched controls (median PCr/ATP 1.72, $\mathrm{p}=\mathrm{ns}$; Figure 1). In patients, those scanned in AF had lower $\operatorname{LVEF}(54 \pm 10$ vs $66 \pm 7 \%$, p <
0.001 ), but not energetics (median PCr/ATP 1.74 vs 1.84 , p $=0.66)$, compared to those scanned in SR.

Early post-ablation, recovery of SR from AF was associated with significant improvement in LVEF compared to patients in SR during both the pre-ablation and early post-ablation scans $(+7 \pm 10$ vs $-0.2 \pm 7 \%, \mathrm{p}=0.02)$. Late post-ablation, LVEF in patients $(63 \pm 8 \%)$ remained impaired compared to matched controls $(\mathrm{p}=0.012)$. Myocardial energetics were 
Spiral flow: where the IVC and SVC combine in a spiral, iv) Complex flow: involving multiple vortices arising from the IVC and SVC flow. (B)-

also unchanged post-ablation (median PCr/ATP 1.74 vs 1.82, $\mathrm{p}=0.39$ ), irrespective of ablation success.

Conclusions Even 'lone' AF is associated with LV dysfunction and impaired myocardial energetics. LV function partially improves with recovery of SR early post-ablation, indicating an acute haemodynamic effect. However, ablation fails to normalise LV function or reverse energetic impairment at later follow-up. These novel findings suggest that human AF may be the consequence (rather than the cause) of an occult cardiomyopathic process that develops with ageing and risk factors. Comprehensive therapeutic strategies that target and reverse this phenotype may reduce $\mathrm{AF}$ recurrence and improve clinical outcomes.

\section{Valve Disease/Pericardial Disease/ Cardiomyopathy}

\section{COMPLICATED INFECTIVE AORTIC ENDOCARDITIS: COMPARISON OF DIFFERENT SURGICAL STRATEGIES}

Miriam Silaschi*, Niki Nicou, Ranjit Deshpande, Max Baghai, Rafal Dworakowski, Olaf Wendler. King's College Hospital; *Presenting Author

\subsection{6/heartjnl-2016-309890.139}

Introduction Infective endocarditis (IE) of the aortic valve complicated by root involvement or an infected prosthesis is a life-threatening condition. While homografts have been promoted previously for treatment, root replacement using stentless bioprostheses (SBP) is an attractive alternative. Still, there

\begin{tabular}{|c|c|c|c|c|}
\hline & $\begin{array}{l}\text { Healthy Controls ( } \mathrm{n} \\
=25 \text { ) }\end{array}$ & $\begin{array}{l}\text { Matched Controls } \\
(n=18)\end{array}$ & $\begin{array}{l}\text { AF Patients ( } \\
=53 \text { ) }\end{array}$ & $\begin{array}{l}\mathrm{p} \\
\text { value }\end{array}$ \\
\hline Age (years) & $51(43-58)$ & $66(63-68)$ \# & $65(57-70)$ \# & $<0.001$ \\
\hline Male (\%) & 56 & 78 & 80 & 0.162 \\
\hline BMI $\left(\mathrm{kg} / \mathrm{m}^{2}\right)$ & $25.7(22-28)$ & $27.4(26-30)$ & $27.2(24-31)^{\#}$ & 0.027 \\
\hline $\begin{array}{l}\text { Resting pulse } \\
\text { (bpm) }\end{array}$ & $60(54-66)$ & $66(60-71)$ & $64(54-80)$ & 0.177 \\
\hline $\mathrm{SBP}(\mathrm{mmHg})$ & 117 (110-125) & $143(125-157)$ \# & $130(124-145)$ \# & $<0.001$ \\
\hline $\mathrm{DBP}(\mathrm{mmHg})$ & $70.9+/ - \pm 10$ & $81.9 \pm 12^{\#}$ & $81.3 \pm 11^{\#}$ & 0.001 \\
\hline
\end{tabular}

P value quoted from one-way ANOVA, Kruskal-Wallis test or Chi-square as appropriate. \# denotes $p<0.05$ versus healthy controls. No statistically significant differences were detected between matched controls and AF patients on post-hoc testing.

is ongoing debate about the optimal substitute in this setting. We compare outcomes of treatment with mechanical prostheses (MP), stented prostheses (SP) and SBP.

Methods Our in-hospital database was explored for patients treated surgically for aortic IE (2000-2015). Valve replacements with homografts were excluded $(\mathrm{n}=12)$. A total of 187 patients received MP $(\mathrm{n}=45)$, SP $(\mathrm{n}=112)$ or SBP ( $=30$ ). All patients with complicated IE (prosthetic valve endocarditis (PVE) or root involvement, $n=77$ ) were included as study cohort. The group was divided according to substitute received (MP $(\mathrm{n}=16)$, SP $(\mathrm{n}=36)$ and SBP $(\mathrm{n}=$ 25)). We analysed short-, long-term- and event-free-survival (100\% complete).

Results SBP and SP patients were older (SBP: $57.8 \pm 15.9 \mathrm{yrs}$, MP: $41.7 \pm 12.4 y r s, S P: 59.7 \pm 15.1 y r s, p<0.01)$. SBP patients suffered more often from PVE $(64.0 \%$ in SBP vs. $18.7 \%$ in $\mathrm{MP}$ and $36.1 \%$ in SP, $\mathrm{p}=0.01$ ), and showed more often root involvement $(100 \%$ in SBP vs. $93.7 \%$ in MP and $83.3 \%$ in SP, $\mathrm{p}=0.08)$. MP patients tended to have a higher rate of active intravenous drug use (SBP: 4.0\%, MP: 25.0\%,
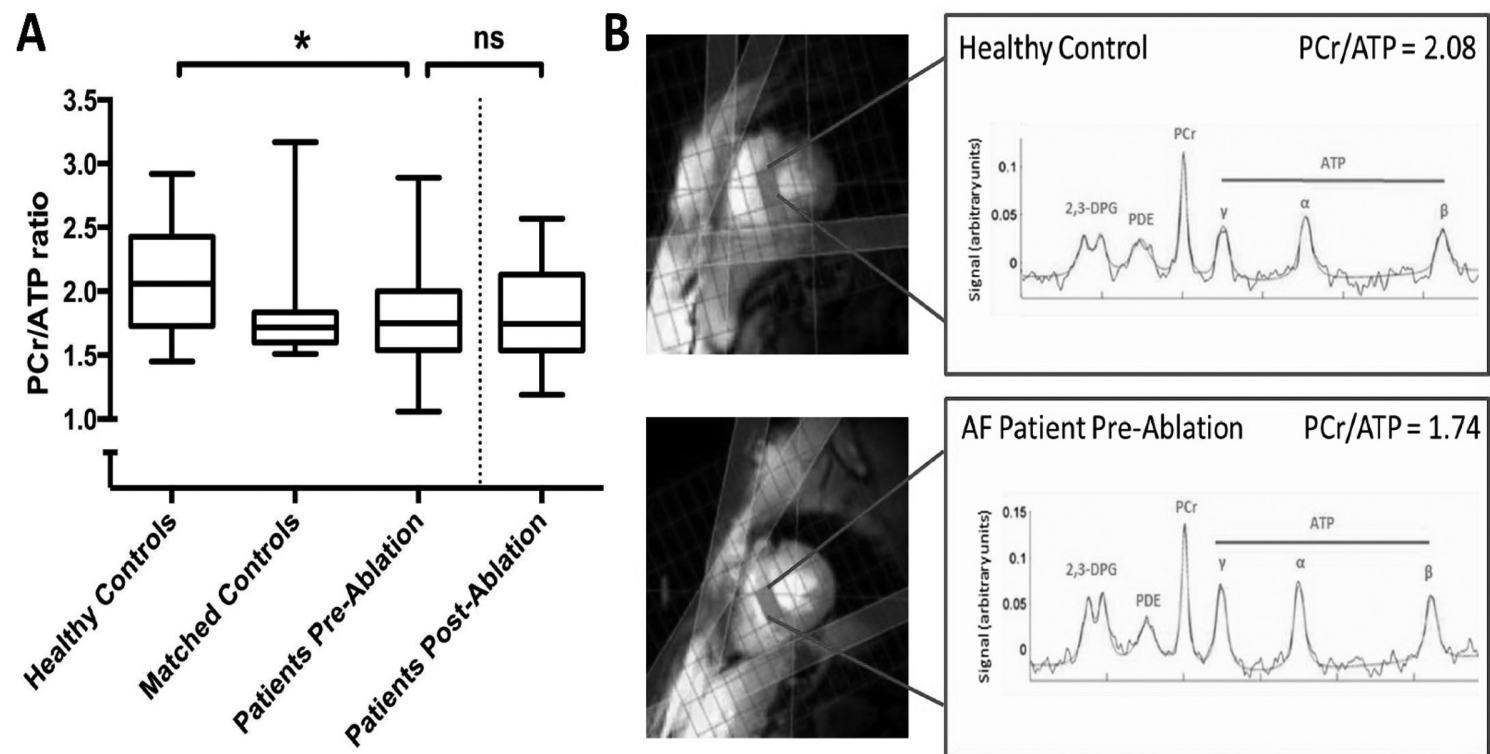

Abstract 138 Figure 1 (A) Myocardial energetics is impaired in patients compared to healthy controls, but not matched controls. (B) Representative 31P spectra from a mid-ventricular septal voxel in a healthy control (PCr/ATP ratio 2.08) and an AF patient pre-ablation (PCr/ATP ratio 1.74). PCr/ATP = ratio of area under phosphocreatine peak to average area under the three ATP peaks. Kruskal-Wallis test with post-hoc pairwise comparison adjusted for multiple testing (healthy controls, matched controls, patients pre-ablation) and separate related-samples Wilcoxon Signed Rank Test (patients pre-ablation and patients post-ablation). *indicates $p<0.05$; centre line indicates median, box indicates interquartile range and error bars indicate range 